\title{
Doxorubicin-loaded PEG-PCL copolymer micelles enhance cytotoxicity and intracellular accumulation of doxorubicin in adriamycin-resistant tumor cells
}

\author{
This article was published in the following Dove Press journal: \\ International Journal of Nanomedicine \\ 9 September 2011 \\ Number of times this article has been viewed
}

\begin{abstract}
Yuan-Yuan Diao 1,2,*
Hao-Ying $\mathrm{Li}^{3, *}$

Ying-Hua $\mathrm{Fu}^{4}$

Min Han'

Yu-Lan Hu'

Hong-Liang Jiang ${ }^{5}$

Yasuo Tsutsumi ${ }^{6}$

Qi-Chun $\mathrm{Wei}^{7}$

Da-Wei Chen ${ }^{2}$

Jian-Qing Gao'

'Institute of Pharmaceutics, College of Pharmaceutical Sciences, Zhejiang

University, Hangzhou; ${ }^{2}$ School of

Pharmacy, Shenyang Pharmaceutical

University, Shenyang; ${ }^{3}$ Biomanufacturing

Research Centre, Department of

Mechanical and Electrical Engineering,

Soochow University, Suzhou;

${ }^{4}$ Department of Pharmaceutics, Jiaxing

University College of Medicine, Jiaxing;

${ }^{5}$ Department of Polymer Science

and Engineering, Zhejiang University,

Hangzhou, People's Republic of China;

${ }^{6}$ Graduate School of Pharmaceutical

Sciences, Osaka University, Osaka, Japan;

${ }^{7}$ Department of Radiation Oncology,

Ministry of Education Key Laboratory

of Cancer Prevention and Intervention,

Zhejiang University School of Medicine,

Hangzhou, People's Republic of China

*These authors contributed equally to this work
\end{abstract}

Correspondence: Jian-Qing Gao; Min Han Institute of Pharmaceutics, College of Pharmaceutical Sciences,

Zhejiang University, Hangzhou

310058 , People's Republic of China

Tel/fax +86 57l 88208437

Email gaojianqing1029@yahoo.com.cn;

hanmin2@zju.edu.cn
Background: Multidrug resistance remains a major obstacle to successful cancer chemotherapy. Some chemical multidrug resistance inhibitors, such as ciclosporin and verapamil, have been reported to reverse resistance in tumor cells. However, the accompanying side effects have limited their clinical application. In this study, we have developed a novel drug delivery system, ie, a polyethyleneglycol-polycaprolactone (PEG-PCL) copolymer micelle encapsulating doxorubicin, in order to circumvent drug resistance in adriamycin-resistant K562 tumor cells.

Methods: Doxorubicin-loaded diblock copolymer PEG-PCL micelles were developed, and the physicochemical properties of these micelles, and accumulation and cytotoxicity of doxorubicin in adriamycin-resistant K562 tumor cells were studied.

Results: Doxorubicin-loaded micelles were prepared using a solvent evaporation method with a diameter of $36 \mathrm{~nm}$ and a zeta potential of $+13.8 \mathrm{mV}$. The entrapment efficiency of doxorubicin was $48.6 \% \pm 2.3 \%$. The micelles showed sustained release, increased uptake, and cellular cytotoxicity, as well as decreased efflux of doxorubicin in adriamycin-resistant K562 tumor cells. Conclusion: This study suggests that PEG-PCL micelles have the potential to reverse multidrug resistance in tumor cells.

Keywords: doxorubicin, polyethylene glycol, polycaprolactone, adriamycin-resistant K562 tumor cells, multidrug resistance, micelles

\section{Introduction}

Cancer is one of the leading causes of morbidity and accounts for a quarter of all deaths around the world. Chemotherapy is one of the most commonly used treatments for cancer, but chemotherapy resistance often limits its efficacy. One of the most frequent phenotypes of multidrug resistance is overexpression in tumor cells of P-glycoprotein, a $170 \mathrm{kDa}$ multidrug resistance 1 gene product. ${ }^{1-3}$ P-glycoprotein can actively pump out its substrates, resulting in reduced intracellular accumulation of certain drugs and decreased therapeutic efficacy. One approach to increasing drug accumulation in cells is to envelop the antitumor drug into nanoscale vehicles to escape recognition by P-glycoprotein.

Micelles formed by amphiphilic block copolymers have attracted significant attention in the medical field due to their various advantages for drug delivery, ${ }^{4}$ which include enhanced solubilization, ${ }^{5}$ stabilization, and delivery of challenging agents. ${ }^{6,7}$ Polymeric micelles are able to accommodate antitumor drugs in their hydrophobic core and thus exceed the solubility limit of hydrophobic drugs. ${ }^{8}$ Furthermore, the hydrophilic corona in micelles 
provides a stabilizing interface between their inner core and the aqueous environment, which help drugs to stay in the circulation over a certain length of time, and hence improve the therapeutic efficacy of the enveloped drugs. ${ }^{67,9}$ Furthermore, the nanosize of polymeric micelles can enhance drug accumulation in solid tumors by prolonging circulation of the drug in the blood, as well as having enhanced permeability and retention effects. ${ }^{10}$ In recent years, more and more studies have shown the potential of micelles to reverse multidrug resistance in tumors. ${ }^{11}$ Several studies have been performed to envelop P-glycoprotein substrates in polymeric micelles or nanoparticles to circumvent drug resistance. ${ }^{12}$ Many amphiphilic agents have been found to be able to enhance cellular accumulation of anticancer drugs, resulting in sensitization of cells overexpressing P-glycoprotein. A good example of this is the Pluronic ${ }^{\circledR}$ family composed of polyethylene oxide-polypropylene oxide-polyethylene oxide triblock copolymers. ${ }^{13,14}$

Doxorubicin, an anthracycline antibiotic, plays an important role in the treatment of leukemia. However, its effects are greatly impaired by multidrug resistance in leukemia cells. ${ }^{15}$ In the present study, we prepared a novel type of doxorubicin-loaded micelle, and demonstrated that these micelles improved the cytotoxicity of doxorubicin by changing the intracellular kinetics of doxorubicin in adriamycinresistant K562 tumor cells.

\section{Materials and methods Materials}

Doxorubicin hydrochloride was obtained from Zhejiang Hisun Pharmaceutical Co Ltd (Taizhou, China). Monomethoxycapped polyethylene glycol (MPEG), and $\xi$-caprolactone was provided by Sigma Aldrich (St Louis, MO). The P-glycoprotein-overexpressing human leukemia cell line. Adriamycin-resistant K562 tumor cells and their P-glycoprotein-nonexpressing counterparts, K562 cells, were generous gifts from the Second Affiliated Hospital of Zhejiang University School of Medicine, Hangzhou, China. RPMI1640 medium and fetal bovine serum were purchased from PAA Laboratories GmbH (Pasching, Austria). MTT was from Shanghai Sangon Biological Engineering Technology and Service Co Ltd (Shanghai, China). $\mathrm{CH}_{2} \mathrm{Cl}_{2}$, triethylamine, and other reagents were of analytical grade and supplied by Huadong Medical (Hangzhou, China).

\section{Synthesis and characteristics of diblock copolymers of PEG-PCL}

The PEG-PCL diblock copolymers were synthesized by an already reported method with minor modifications. ${ }^{16,17}$
The chemical structure is shown in Figure 1A. Briefly, ring-opening polymerization of $\xi$-caprolactone was induced by using MPEG as the macroinitiator and calcium ammoniate as the catalyst. The product was then purified by precipitating twice into cold methanol from $\mathrm{CH}_{2} \mathrm{Cl}_{2}$ solution, and was finally dried under vacuum at $40^{\circ} \mathrm{C}$. The molecular weight of the segments in the diblock copolymer was determined by the intensity of the terminal proton signal of MPEG at 3.39 ppm and the methylene proton signal of PCL at $2.31 \mathrm{ppm}$ using ${ }^{1} \mathrm{H}$ nuclear magnetic resonance spectroscopy. The weight ratio of the PEG-PCL repeated units calculated from the integral values of characteristic peaks was 3.19 (PCL 4222-PEG 4225; EG/CL 3.19). Molecular weight obtained by gel permeation chromatography measurements was about 17188 Da (Figure 1B).

\section{Preparation of doxorubicin-loaded PEG-PCL micelles}

Polymeric micelles containing doxorubicin hydrochloride were prepared by the solvent evaporation method. ${ }^{18,19}$ Briefly, doxorubicin hydrochloride $3 \mathrm{mg}$ was dissolved in $\mathrm{CH}_{2} \mathrm{Cl}_{2}$ $2 \mathrm{~mL}$ in the presence of a triple molar ratio of triethylamine, and stirred at $500 \mathrm{rpm}$ on a magnetic stirrer for 4 hours at room temperature. Then PEG-PCL copolymer $10 \mathrm{mg}$ was added into the solvent and vortexed until it was totally dissolved. Afterwards, the organic solution containing the drug and the polymer were added to $20 \mathrm{~mL}$ distilled water under vigorous ultrasonication. The mixture was first stirred vigorously at $1000 \mathrm{rpm}$ for 1 hour and then slowly at $200 \mathrm{rpm}$ overnight to allow slow evaporation of $\mathrm{CH}_{2} \mathrm{Cl}_{2}$ and formation of the micelles. The residual $\mathrm{CH}_{2} \mathrm{Cl}_{2}$ was completely removed by vacuum distillation using a rotary evaporator. The micelle solution, concentrated to $5 \mathrm{~mL}$, was filtered with a syringe filter (pore size $0.22 \mu \mathrm{m}$ ) to eliminate the polymer and doxorubicin aggregates. All the procedures were carried out under light protection.

\section{Characterization of micelles}

The morphologies of the micelles were studied using transmission electron microscopy (Morgagni 268 D; FEI, Eindhoven, The Netherlands), and dynamic light scattering (Malvern Nano ZS ${ }^{\circledR}$, Malvern Instruments Ltd, Worcestershire, UK) was used to determine the diameter. The zeta potential was measured using a Zetasizer Nano-Z (Malvern Instruments Ltd). Drug entrapment efficiency was calculated as the ratio between the amount of drug incorporated into the micelles and the total amount of drug. Free doxorubicin was separated by an ultrafiltration membrane (Millipore, Billerica, MA) 
A

\section{$\mathrm{CH}_{3}\left(\mathrm{OCH}_{2} \mathrm{CH}_{2}\right)_{x} \mathrm{O}\left[\mathrm{O}\left[\mathrm{C}\left(\mathrm{CH}_{2}\right)_{5}\right)-y\right.$}

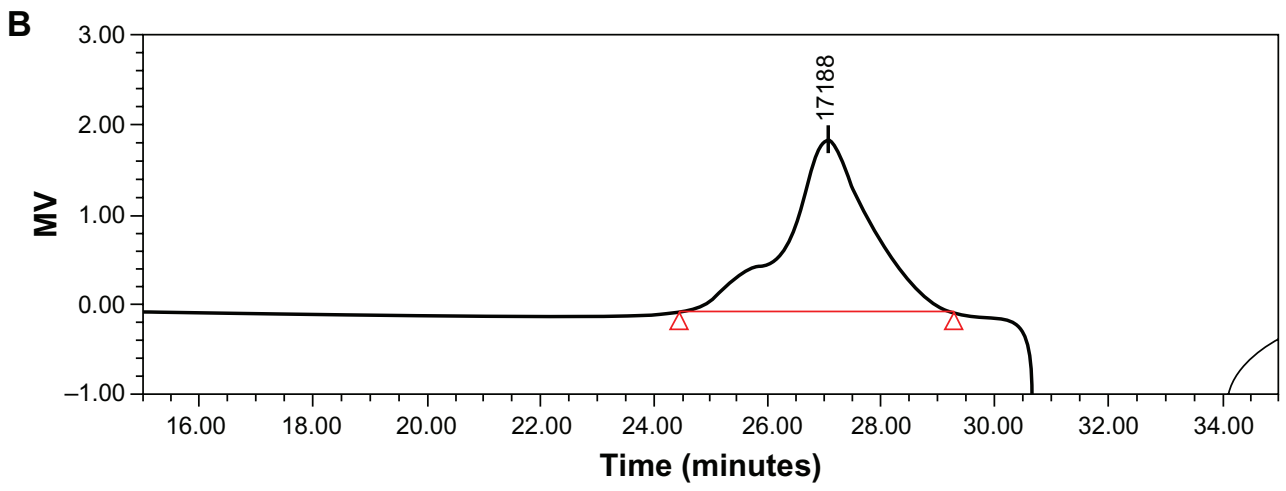

Figure I (A) Chemical structure and (B) molecular weight of diblock copolymer polyethylene glycol-polycaprolactone. Molecular weight was determined by gel permeation chromatography measurements.

with a molecular weight cutoff of 50,000 Da, and doxorubicin content was measured by high-performance liquid chromatography (Agilent 1100; Agilent Technologies, Inc, Santa Clara, CA).

\section{In vitro release of doxorubicin from polymer micelles}

Micelle solution $1 \mathrm{~mL}$ was transferred into dialysis bags (molecular weight cutoff 12,000 Da; Spectrum Laboratories, Rancho Dominguez, CA) and placed in $10 \mathrm{~mL}$ of phosphatebuffered saline solution with addition of Tween-80 $0.1 \%$. A drug release study was performed at $37^{\circ} \mathrm{C}$ in a thermostatic shaker at a rate of $75 \mathrm{rpm}$. Three milliliters of the buffered solution outside the dialysis bag were removed for analysis at predetermined intervals, and replaced with fresh buffer solution. Doxorubicin concentration was determined using the high-performance liquid chromatography method, as described above.

\section{Cytotoxicity assay}

Cells were cultured in growth medium consisting of RPMI-1640 supplemented with 10\% fetal bovine serum and $1 \%$ penicillin/streptomycin. Subculture was performed every other day. Adriamycin-resistant K562 tumor cells were maintained in a complete RPMI-1640 medium containing doxorubicin $1 \mu \mathrm{g} / \mathrm{mL}$ at $37^{\circ} \mathrm{C}$ in a humidified atmosphere of $5 \% \mathrm{CO}_{2}$. The cells were cultured for 2 weeks in drug-free medium prior to their use. To examine the levels of resistance in the selected cell lines, the cells were seeded in 96-well plates at a density of $10^{4}$ cells/well, and allowed to grow overnight. A specified concentration of doxorubicin-free or doxorubicin-loaded micelles was added into the culture medium on the following day. Control experiments were conducted in parallel by adding $20 \mu \mathrm{L}$ of sterile water, and followed by 72 hours of cultivation at $37^{\circ} \mathrm{C}$ in a humidified $5 \% \mathrm{CO}_{2}$ atmosphere. Meanwhile, the cytotoxicity of the physical mixture of copolymer and doxorubicin solution was also determined. The cytotoxic activity of doxorubicin was evaluated using a standard MTT assay. All experiments were performed in triplicate. Absorbance at $560 \mathrm{~nm}$ was determined using a microplate reader. The results were calculated using the following equation:

\section{Cytotoxicity $\%=($ one experiment group $/$ control}

$$
\text { group) } \times 100 \%
$$

\section{Determination of cellular uptake}

In order to evaluate the accumulation of doxorubicin in adriamycin-resistant K562 tumor cells, a cell suspension containing different concentrations of doxorubicin solution or doxorubicin-loaded micelles, was seeded at a density of $2 \times 10^{6}$ cells $/ \mathrm{mL}$ in $2 \mathrm{~mL}$ of growth medium. The plates were incubated for two hours. The culture medium was then removed. The cells were washed twice using ice-cold phosphate-buffered saline to remove noninternalized doxorubicin, and subsequently resuspended in $1 \mathrm{~mL}$ of water. The cells were treated by probe-type ultrasonication to obtain the cell lysate. The lysate was centrifuged at 10,000 rpm for 5 minutes in a microcentrifuge (Eppendorf AG, Hamburg, Germany) to remove the cell debris. The supernatant was analyzed using 
high-performance liquid chromatography (Waters 474) equipped with a 474 multiwavelength fluorescence detector (excitation $497 \mathrm{~nm}$ and emission $555 \mathrm{~nm}$ ). Calibration curves of known concentrations of doxorubicin in water were used for quantification. The mobile phase used was acetonitrile/0.015 $\mathrm{M} \mathrm{NaH}_{2} \mathrm{PO}_{4}$ solution ( $\mathrm{pH} 4.5,30: 70$ ), and the flow rate was kept at $1 \mathrm{~mL} /$ minute.

\section{Confocal laser scanning microscopy}

After adriamycin-resistant K562 tumor cells were seeded in 12-well plates at a density of $5 \times 10^{4}$ cells/well for 24 hours, the cell culture medium was replaced by $1 \mathrm{~mL}$ of phosphate-buffered saline containing doxorubicin solution or doxorubicin-loaded micelle solution (doxorubicin concentration $24 \mu \mathrm{g} / \mathrm{mL}$ ) for 30 minutes, 2 hours, and 2 hours. The cells were then examined by confocal laser scanning microscopy (Carl Zeiss, Shanghai Co Ltd, China) with an excitation wavelength of $458 \mathrm{~nm}$.

\section{Statistical analysis}

All results were analyzed using Student's $t$-test with a statistical significance level of $P<0.05$.

\section{Results}

\section{Properties of drug-loaded micelles}

The shape and morphology of the micelles observed by transmission electron microscopy is shown in Figure 2 (left). Presence of the micelles is shown by light spherical entities surrounded by dark staining. It can be seen that the diblock copolymers formed spherical particles with a small diameter and a narrow size distribution. Dynamic light scattering studies indicated that the mean diameter was around $36 \mathrm{~nm}$ (Figure 2, right) with a zeta potential of $13.8 \mathrm{mV}$. The content of doxorubicin in the micelles was verified as $12.6 \%$. Doxorubicin was encapsulated in the hydrophobic segment of the copolymer. The percentage entrapment efficiency depended largely on the drug/polymer ratio, and reached the highest value $(48.15 \%)$ when the drug and copolymer weight ratio was 3:10 (Figure 3). This relatively low entrapment efficiency of micelles produced by the solvent evaporation method is probably due to slow leakage of doxorubicin into the dispersion medium during the stirring procedure. ${ }^{20}$

\section{Envelopment of doxorubicin in micelles induced sustained release of doxorubicin in vitro}

As shown in Figure 4, doxorubicin was released from micelles with an initial burst release, followed by a phase of slow release, and the percentage of release over 30 hours was around $70 \%$. In comparison, doxorubicin solution was released completely within 2 hours. This indicates that envelopment of doxorubicin in the micelles resulted in significantly sustained release of the drug, which is beneficial for drug action.

\section{Envelopment of doxorubicin in micelles enhanced cytotoxicity and accumulation in adriamycin-resistant $\mathrm{K} 562$ tumor cells}

Figure 5 shows the cytotoxicity of doxorubicin-loaded micelles and doxorubicin solution in K562 cells (left) and adriamycin-resistant K562 tumor cells (right). Viability was
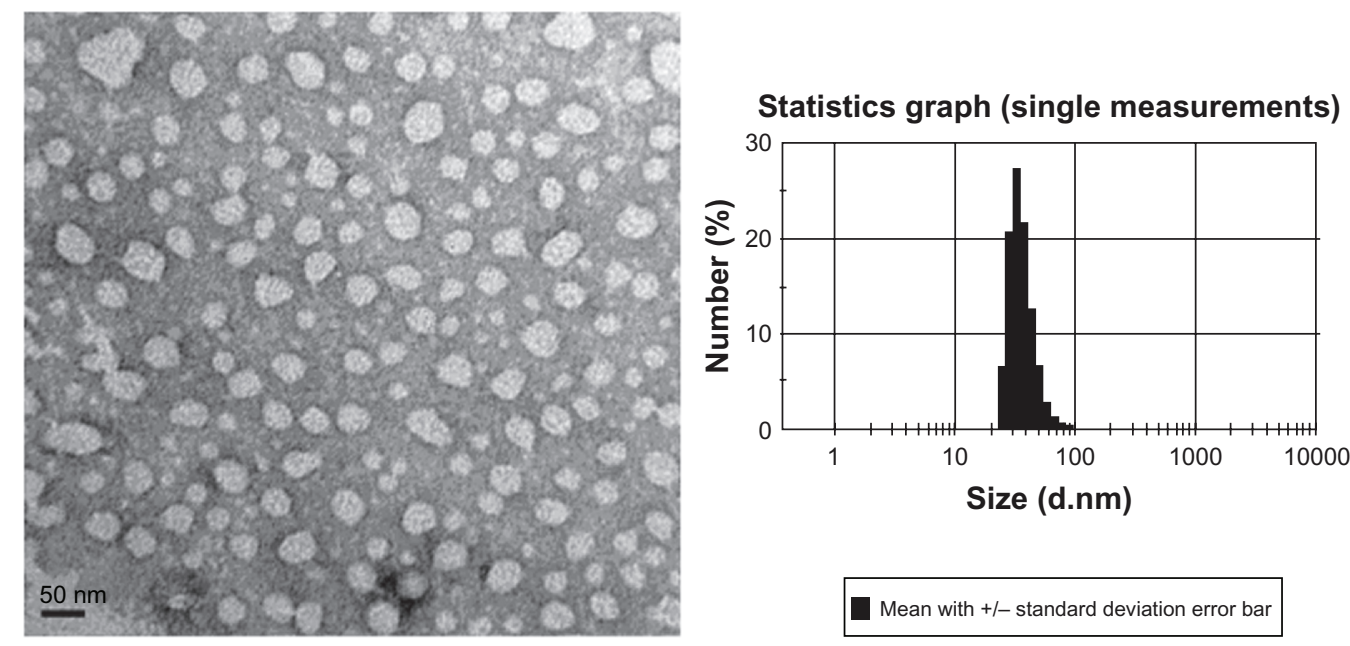

Figure 2 Morphology and size distribution of doxorubicin-loaded polyethyleneglycol-polycaprolactone micelles. The morphologies of the micelles were studied using transmission electron microscopy (left). Dynamic light scattering was used to determine the diameter of micellar doxorubicin. 


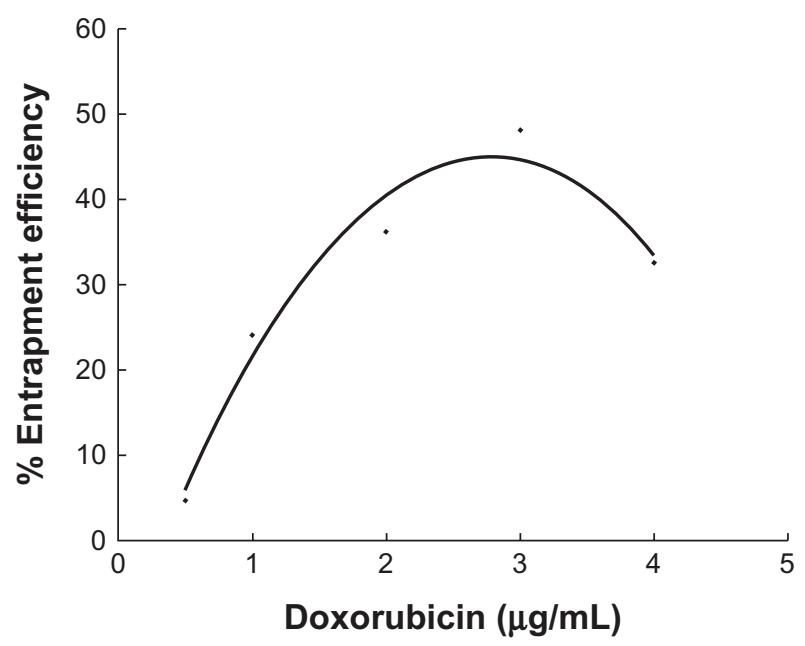

Figure 3 Entrapment efficiency of the doxorubicin in the micelle that was influenced by the quantity of added drug. The drug entrapment efficiency was the ratio (percentage) of the amount of drug incorporated into micelles to the total amount of the drug.

dramatically decreased in the K562 cells, and no significant differences were observed after incubating the cells with either doxorubicin solution or doxorubicin-loaded micelles for three days at doxorubicin concentrations of $3-12 \mu \mathrm{g} / \mathrm{mL}$ $(P>0.05)$. In the adriamycin-resistant $\mathrm{K} 562$ tumor cells, notably enhanced cytotoxicity was induced by drug-loaded micelles when compared with doxorubicin solution at a concentration of $6-12 \mu \mathrm{g} / \mathrm{mL}$. At the same time, the physical mixture of polymer and doxorubicin showed the same cytotoxicity as the doxorubicin solution. Internalization of micellar doxorubicin and free doxorubicin in the adriamycinresistant K562 tumor cells was examined using fluorescent high-performance liquid chromatography. After two hours of incubation, envelopment of doxorubicin in the micelles significantly enhanced cellular accumulation of the drug, especially at an initial drug concentration of $6 \mu \mathrm{g} / \mathrm{mL}$ and $12 \mu \mathrm{g} / \mathrm{mL}$ (Figure 6).

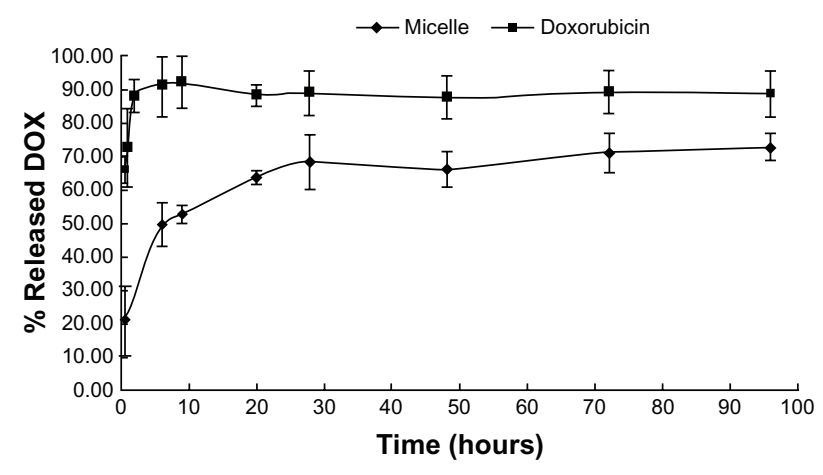

Figure 4 In vitro doxorubicin release profiles from doxorubicin-loaded micelles and doxorubicin solution. A drug release study was performed at $37^{\circ} \mathrm{C}$ under shaking (75 rpm) which was monitored using a dialysis bag containing phosphate-buffered saline with addition of $0.1 \%$ Tween- 80 as a sink solution at $\mathrm{pH} 7.4$.

\section{Distribution of doxorubicin and drug-loaded micelles in multidrug-resistant $\mathrm{K} 562$ cells}

The distribution of doxorubicin solution and doxorubicinloaded micelles in multidrug-resistant K562 cells was studied by confocal laser scanning microscopy. Figure 7 showed that after 30 minutes of cell incubation with free doxorubicin, strong fluorescence was observed in the cells, whereas those cells exposed to free drug for two hours showed very weak fluorescence, and eight hours later, even less doxorubicin was observed in the cells. In contrast with the cells treated using doxorubicin-free solution, cells incubated with doxorubicin-loaded PEG-PCL micelles generated much weaker fluorescence at the time of 30 minutes, and a much stronger fluorescence after 8 hours of treatment. At 30 minutes, fluorescence in the cells was much weaker than that of the free doxorubicin. However, after 2 hours of incubation, doxorubicin emitted strong fluorescence from the cells, and after 8 hours, a lot of doxorubicin was still found in the cells.

\section{Discussion}

The smaller the nanoparticle size, the better its stability in aqueous solution. ${ }^{19}$ In the current study, we obtained micelles with a particle size smaller than the reported values. ${ }^{21,22}$ This may be attributed to longer PEG chains and the lower molecular weight of the copolymer. The diameter of micelles depends largely on the hydrophilic/hydrophobic chain ratio, and higher PEG/PCL chain ratios produce smaller micelle diameters. Furthermore, a long PEG chain on the micelle surface could improve the stability of micelles because of a much lower critical micelle concentration. ${ }^{19}$ The polymer we synthesized has a much longer PEG chain compared with its PCL chain, which resulted in a much smaller particle size and improvement in micelle stability.

An in vitro release experiment indicated that envelopment of doxorubicin in micelles significantly delayed drug release, which could both enhance drug stability and prolong drug action. The initial burst which happened within the first few hours of incubation may be attributed to a relatively small amount of drug at the interface between the micelle hydrophobic core and hydrophilic corona. ${ }^{23,24}$ Several investigations have demonstrated that a sustained pattern of release of antitumor drug from the carrier would enhance tumorsuppressive activity both in vitro ${ }^{24}$ and in vivo. ${ }^{25}$

Figure 5 shows that micellar doxorubicin had much greater cytotoxicity than doxorubicin solution in multidrugresistant K562 cells, indicating the potential of micellar doxorubicin to reverse multidrug resistance. The ability of 

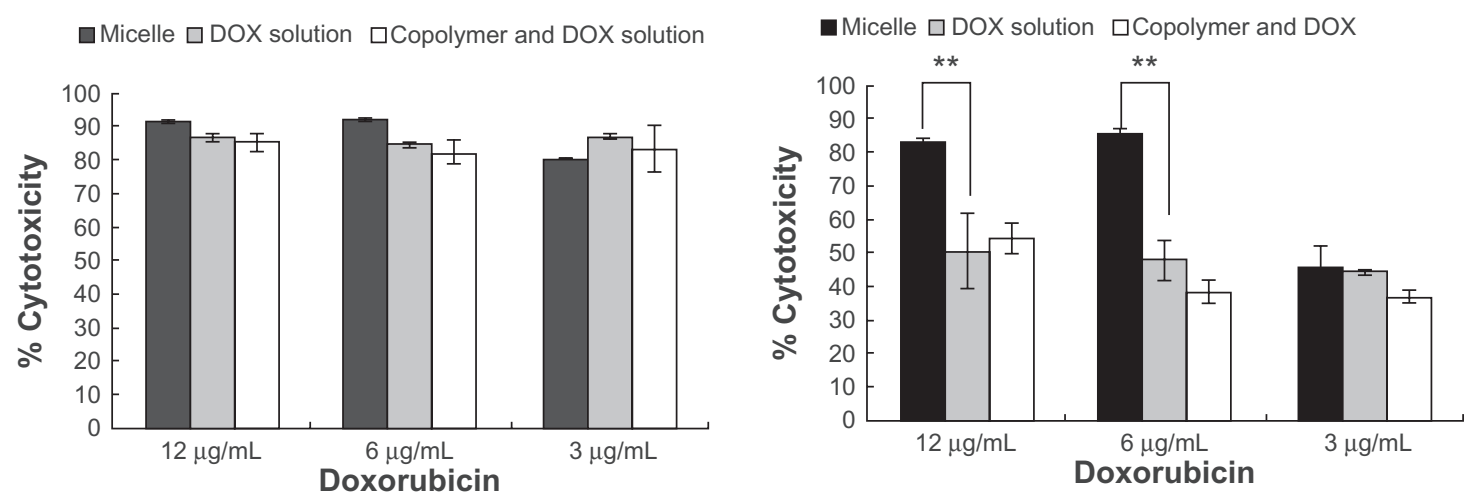

Figure 5 Cytotoxicity determined by MTT assay in (A) K562 cells and (B) adriamycin-resistant K562 tumor cells. Cells were incubated with doxorubicin-loaded micelles, doxorubicin solution, and a physical mixture of the copolymer and doxorubicin for 72 hours. The doxorubicin concentration is varied.

Notes: Error bars represent the standard deviations $(\mathrm{n}=3)$ : $* P<0.05 ; * * P<0.01$.

micelles to enhance the cytotoxicity of the antitumor drug and reverse multidrug resistance has also been demonstrated by other reports. For example, Wang et $\mathrm{al}^{26}$ reported that micellar P105 and P105/L101 paclitaxel could overcome multidrug resistance in adriamycin-resistant $\mathrm{MCF}-7$ tumor cells. The higher cytotoxic activity of doxorubicin-loaded micelles over free doxorubicin implies that doxorubicin encapsulated in PEG-PCL could elevate the intracellular concentration of doxorubicin through different transport process from doxorubicin solution, which was supported by the intracellular uptake study we performed. Yi et $\mathrm{al}^{27}$ also reported that a doxorubicin-loaded amphiphilic copolymer micelle composed of MPEG, poly 2-(dimethylamino) ethyl methacrylate, and poly 2-(diethylamino)ethyl methacrylate showed 38.1 times higher activity in adriamycinresistant MCF-7 tumor cells than free doxorubicin after three days of incubation. Furthermore, polyethylene

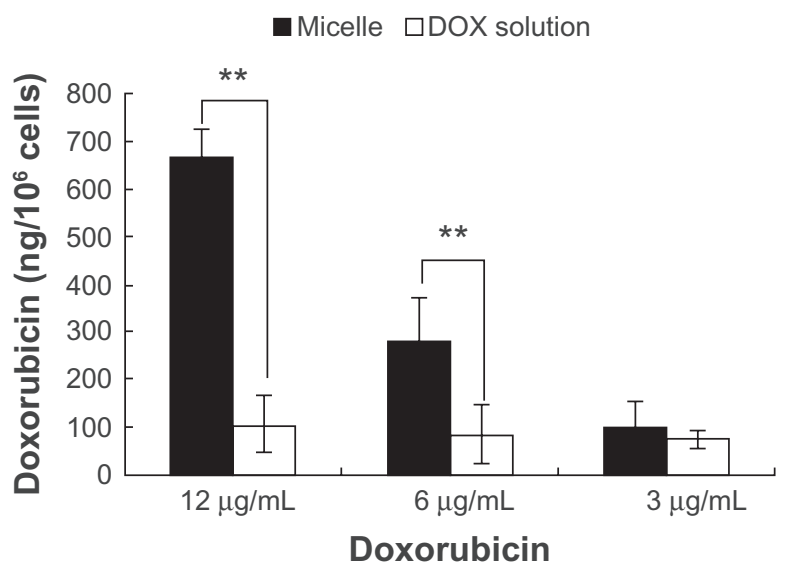

Figure 6 The intracellular doxorubicin amount in adriamycin-resistant K562 tumor cells. Doxorubicin uptake into the adriamycin-resistant K562 tumor cells after incubation with different drug concentrations of micellar doxorubicin and doxorubicin solution for 2 hours.

Notes: $* P<0.05 ; * * P<0.01$. glycol-block-polypropylene glycol-block-polyethylene glycol micelles loaded with doxorubicin could interact with multidrug resistance cancer cells, resulting in drastic sensitization of these tumor cells. ${ }^{13}$ However, the physical mixture of polymer and doxorubicin did not show enhanced cytotoxicity compared with the doxorubicin solution, indicating that the cytotoxicity of the micelles was not induced or enhanced by polymer materials.

In the present study, we observed that doxorubicin entrapped in micelles could enhance drug accumulation in multidrug-resistant K562 cells, which is consistent with the finding that a series of diblock copolymers based on methoxypolyethylene glycol-block-polycaprolactone enhanced cellular accumulation of another P-glycoprotein substrate, rhodamine-123, in Caco-2 cells. ${ }^{28,29}$ The increased accumulation of doxorubicin in adriamycin-resistant K562 tumor cells indicated that doxorubicin-loaded micelles could enhance
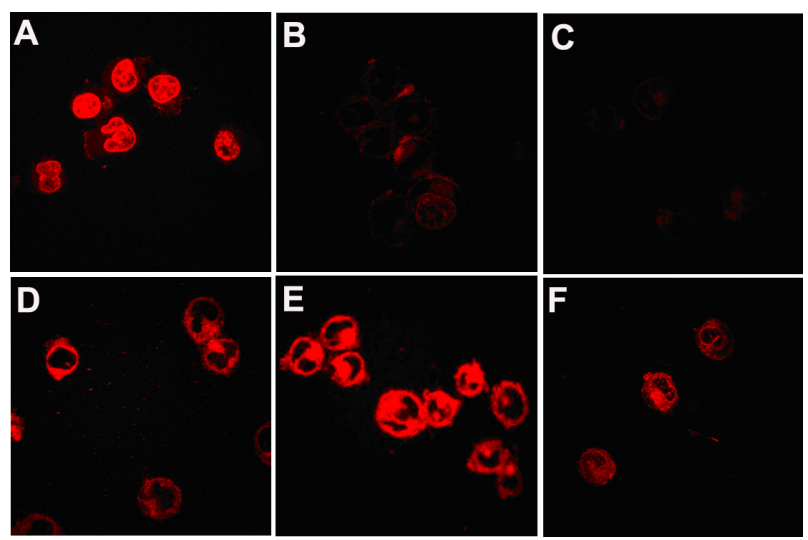

Figure 7 Distribution of doxorubicin in the multidrug-resistant $\mathrm{K} 562$ tumor cells. It was observed under confocal microscopy after incubating multidrug-resistant K562 tumor cells with free doxorubicin for (A) 0.5 hours, (B) 2 hours, (C) 8 hours, and doxorubicin-loaded copolymer polyethylene glycol-polycaprolactone micelles for (D) 30 minutes, (E) 2 hours, and (F) 8 hours. 
the sensitivity and uptake of adriamycin-resistant K562 tumor cells by doxorubicin, which also hints at the potential of micellar doxorubicin to reverse P-glycoprotein-mediated multidrug resistance.

Confocal laser scanning microscopy results showed that after incubation of multidrug-resistant K562 cells with doxorubicin solution for half an hour, doxorubicin could enter the nucleus of these cells quickly, but less doxorubicin was found in the cells after two hours of incubation, and little doxorubicin was in the cells after eight hours. It has been suggested that free doxorubicin can also enter into drug-resistant cells at first, but is then pumped out of the cells quickly. As for the micellar doxorubicin, although it enters into the cells at a lower rate than free doxorubicin, it may not be pumped out until 2 or 8 hours. In addition, endocytosis may contribute a lot to the uptake of micellar doxorubicin by adriamycin-resistant K 562 tumor cells, while the migration of free doxorubicin into the cells may mostly rely on a passive diffusion process. This may explain why free doxorubicin entered the cells more quickly than micellar doxorubicin. However, the micelles could be entrapped by endosomes and lysosomes, and thus would escape the efflux by P-glycoprotein.

Moreover, micelles released doxorubicin in a sustained way (Figure 4), and have enhanced cytotoxicity. Similar results were also obtained by Shuai et $\mathrm{al}^{16}$ and Savic et al. ${ }^{30}$ Their studies showed that the patterns of cellular distribution of doxorubicin-loaded MPEG-PCL micelles and free doxorubicin are different in PC12 cells. They demonstrated that micelles are internalized into PC12 cells via an endocytosis mechanism and doxorubicin in endosomes and lysosomes is higher for doxorubicin-loaded MPEG-PCL micelles, while free doxorubicin entered into the cells by diffusion. Allen et $\mathrm{al}^{31}$ also investigated cellular internalization of PCL-b-polyethylene oxide copolymer micelles in PC12 cells, and the internalization process of the micelles was found to fulfill the basic criteria of endocytosis (uptake was time-, temperature-, $\mathrm{pH}$-, and energy-dependent).

Thus, based on the findings from our study and evidence from the literature, we propose that doxorubicin delivered by PEG-PCL copolymer micelle enters into cells via internalization, and intracellular entry of doxorubicin-loaded micelles followed by entrapment in endosomes and lysosomes renders the drug inaccessible to P-glycoprotein. Micellar doxorubicin is released in a controlled manner from the micelle particles as shown in Figure 4, and eventually enters the nucleus where doxorubicin is known to exert its cytotoxicity during DNA synthesis.
In conclusion, biodegradable diblock copolymers of PEG-PCL was synthesized by ring-opening polymerization of $\xi$-caprolactone for effective delivery of doxorubicin, an anticancer drug, into tumor cells. This amphiphilic polymer self-assembled into core shell structural micelles, and encapsulated doxorubicin in the hydrophobic core. Compared with doxorubicin solution, micelle-delivered doxorubicin showed more potent cytotoxicity in adriamycin-resistant K562 tumor cells, and enhanced accumulation of doxorubicin in tumor cells, indicating its ability to reverse multidrug resistance.

\section{Acknowledgment}

This study was financially supported by the Scientific Research Fund of the Ministry of Health-Medical Science Critical Technological Program of Zhejiang Province, China (No. WKJ2008-2-029) the Natural Science Foundation of Zhejiang Province, China (No. Y2110124) and the Research Fund for the Doctoral Program of Higher Education of China (No. 20090101120141).

\section{Disclosure}

The authors report no conflicts of interest in this work.

\section{References}

1. Damiani D, Tiribelli M, Raspadori D, et al. The role of MDR-related proteins in the prognosis of adult acute myeloid leukaemia (AML) with normal karyotype. Hematol Oncol. 2007;25(1):38-43.

2. Gottesman MM, Pastan I, Ambudkar SV. P-glycoprotein and multidrug resistance. Curr Opin Genet Dev. 1996;6(5):610-617.

3. Michieli M, Damiani D, Ermacora A, et al. P-glycoprotein, lung resistance-related protein and multidrug resistance associated protein in de novo acute non-lymphocytic leukaemias: Biological and clinical implications. Br J Haematol. 1999;104(2):328-335.

4. Gou M, Zheng X, Men K, et al. Self-assembled hydrophobic honokiol loaded MPEG-PCL diblock copolymer micelles. Pharm Res. 2009;26(9):2164-2173.

5. Sezgin Z, Yüksel N, Baykara T. Preparation and characterization of polymeric micelles for solubilization of poorly soluble anticancer drugs. Eur J Pharm Biopharm. 2006;64(3):261-268.

6. Adams ML, Lavasanifar A, Kwon GS. Amphiphilic block copolymers for drug delivery. J Pharm Sci. 2003;92(7):1343-1355.

7. Kataoka K, Harada A, Nagasaki Y. Block copolymer micelles for drug delivery: Design, characterization and biological significance. Adv Drug Deliv Rev. 2001;47(1):113-131.

8. Prabaharan M, Grailer JJ, Pilla S, Steeber DA, Gong S. Amphiphilic multi-arm block copolymer based on hyperbranched polyester, poly(L-lactide) and poly(ethylene glycol) as a drug delivery carrier Macromol Biosci. 2009;9(5):515-524.

9. Yokoyama M, Okano T, Sakurai Y, Ekimoto H, Shibazaki C, Kataoka K Toxicity and antitumor activity against solid tumors of micelle-forming polymeric anticancer drug and its extremely long circulation in blood. Cancer Res. 1991;51(12):3229-3236.

10. Koziara JM, Whisman TR, Tseng MT, Mumper RJ. In-vivo efficacy of novel paclitaxel nanoparticles in paclitaxel-resistant human colorectal tumors. J Control Release. 2006;112(3):312-319.

11. Chen JN, Shen Q, Li SS. Progress in the study of drug delivery system based on nanoparticles to overcome multi-drug resistance. Yaо Хие Xиe Bao. 2009;44(4):333-337. Chinese. 
12. Jabr-Milane LS, van Vlerken LE, Yadav S, Amiji MM. Multi-functional nanocarriers to overcome tumor drug resistance. Cancer Treat Rev. 2008;34(7):592-602.

13. Kabanov AV, Batrakova EV, Alakhov VY. Pluronic block copolymers for overcoming drug resistance in cancer. Adv Drug Deliv Rev. 2002;54(5):759-779.

14. Minko T, Batrakova EV, Li S, et al. Pluronic block copolymers alter apoptotic signal transduction of doxorubicin in drug-resistant cancer cells. J Control Release. 2005;105(3):269-278.

15. Chen VY, Posada MM, Zhao L, Rosania GR. Rapid doxorubicin efflux from the nucleus of drug-resistant cancer cells following extracellular drug clearance. Pharm Res. 2007;24(11):2156-2167.

16. Shuai X, Ai H, Nasongkla N, Kim S, Gao J. Micellar carriers based on block copolymers of poly(epsilon-caprolactone) and poly(ethylene glycol) for doxorubicin delivery. J Control Release. 2004;98(3):415-426.

17. Zhang J, Wang LQ, Wang H, Tu K. Micellization phenomena of amphiphilic block copolymers based on methoxy poly(ethylene glycol) and either crystalline or amorphous poly(caprolactone-b-lactide). Biomacromolecules. 2006;7(9):2492-2500.

18. Shuai X, Merdan T, Schaper AK, Xi F, Kissel T. Core-crosslinked polymeric micelles as paclitaxel carriers. Bioconjug Chem. 2004;15(3):441-448.

19. Xu P, Tang H, Li S, et al. Enhanced stability of core-surface crosslinked micelles fabricated from amphiphilic brush copolymers. Biomacromolecules. 2004;5(5):1736-1744.

20. Shi YQ, Qu XJ, Liao YX, et al. Reversal effect of a macrocyclic bisbibenzyl plagiochin $\mathrm{E}$ on multidrug resistance in adriamycin-resistant K562/A02 cells. Eur J Pharmacol. 2008;584(1):66-71.

21. Li B, Moriyama EH, Li F, Jarvi MT, Allen C, Wilson BC. Diblock copolymer micelles deliver hydrophobic protoporphyrin IX for photodynamic therapy. Photochem Photobiol. 2007;83(6):1505-1512.

22. Allen C, Yu Y, Maysinger D, Eisenberg A. Polycaprolactone-bpoly(ethylene oxide) block copolymer micelles as a novel drug delivery vehicle for neurotrophic agents FK506 and L-685, 818. Bioconjug Chem. 1998;9(5):564-572.
23. Okuda T, Kawakami S, Higuchi Y, et al. Enhanced in vivo antitumor efficacy of fenretinide encapsulated in polymeric micelles. Int JPharm. 2009;373(1-2):100-106.

24. Wang J, Wang R, Li LB. Preparation and properties of hydroxycamptothecin-loaded nanoparticles made of amphiphilic copolymer and normal polymer. J Colloid Interface Sci. 2009;336(2):808-813.

25. Elazar V, Adwan H, Bäuerle T, Rohekar K, Golomb G, Berger MR. Sustained delivery and efficacy of polymeric nanoparticles containing osteopontin and bone sialoprotein antisenses in rats with breast cancer bone metastasis. Int J Cancer. 2010;126(7):1749-1760.

26. Wang Y, Yu L, Han L, Sha X, Fang X. Difunctional Pluronic copolymer micelles for paclitaxel delivery: Synergistic effect of folate-mediated targeting and Pluronic-mediated overcoming multidrug resistance in tumor cell lines. Int J Pharm. 2007;337(1-2):63-73.

27. Yi Y, Kim JH, Kang HW, Oh HS, Kim SW, Seo MH. A polymeric nanoparticle consisting of mPEG-PLA-Toco and PLMA-COONa as a drug carrier: Improvements in cellular uptake and biodistribution. Pharm Res. 2005;22(2):200-208.

28. Zastre J, Jackson J, Bajwa M, Liggins R, Iqbal F, Burt H. Enhanced cellular accumulation of a P-glycoprotein substrate, rhodamine-123, by Caco-2 cells using low molecular weight methoxypolyethylene glycolblock-polycaprolactone diblock copolymers. Eur J Pharm Biopharm. 2002;54(3):299-309.

29. Zastre J, Jackson JK, Wong W, Burt HM. Methoxypolyethylene glycolblock-polycaprolactone diblock copolymers reduce P-glycoprotein efflux in the absence of a membrane fluidization effect while stimulating P-glycoprotein ATPase activity. J Pharm Sci. 2007;96(4):864-875.

30. Savic R, Luo L, Eisenberg A, Maysinger D. Micellar nanocontainers distribute to defined cytoplasmic organelles. Science. 2003;300(5619):615-618.

31. Allen C, Yu Y, Eisenberg A, Maysinger D. Cellular internalization of PCL(20)-b-PEO(44) block copolymer micelles. Biochim Biophys Acta. $1999 ; 1421(1): 32-38$.
International Journal of Nanomedicine

\section{Publish your work in this journal}

The International Journal of Nanomedicine is an international, peerreviewed journal focusing on the application of nanotechnology in diagnostics, therapeutics, and drug delivery systems throughout the biomedical field. This journal is indexed on PubMed Central, MedLine, CAS, SciSearch $\AA$, Current Contents ${ }^{\circledR} /$ Clinical Medicine,

\section{Dovepress}

Journal Citation Reports/Science Edition, EMBase, Scopus and the Elsevier Bibliographic databases. The manuscript management system is completely online and includes a very quick and fair peer-review system, which is all easy to use. Visit http://www.dovepress.com/ testimonials.php to read real quotes from published authors. 